\title{
INTEGRATED DIAGNOSIS (RADIOLOGY, PATHOLOGY AND GENETICS): EARLY EXPERIENCE
}

\section{DIAGNÓSTICO INTEGRADO (RADIOLOGÍA, PATOLOGÍA Y GENÉTICA): EXPERIENCIA TEMPRANA}

\section{Pablo Ros}

Department of Radiology. Stony Brook University, Stony Brook, New York

When you become comfortable with uncertainty,

infinite possibilities open in your life.

Eckhart Tolle

\section{INTRODUCTION}

The objectives of this review are two. The first one is for the reader to become familiar with the concept of Integrated Diagnosis (ID), which combines Radiology, Pathology and Genomics into an innovative diagnostic tool. A second objective is to explain how the current computational revolution provides the technological basis for the cross-disciplinary implementation of Integrated Diagnosis. In order to achieve this dual purpose, we describe a Diagnostic Institute model in a United States Academic Medical Center (AMC) (1).

\section{DEFINITION}

Integrated diagnosis is defined as the integration of Radiology, Pathology and Genetics as the three core diagnostic disciplines. These traditionally independent specialties although mutually interdependent have few touching points with clear clinical impact despite the fact they are part of the routine medical care of the majority of patients (1).

\section{BACKGROUND}

A traditional partnership exists for decades around radiologic-pathologic correlation, mainly as a teaching and academic tool to show, to primarily radiology trainees, the underlying pathologic basis for the appearance of lesions. The Registry of Radiologic Pathology was established in 1947 at the Armed Forces Institute of Pathology (AFIP) and until the Institute's closure in 2005 one of its missions was to teach radiologic-pathologic correlation. The AFIP taught thousands of residents from around the world, but mainly from practically all the Diagnostic Radiology North American teaching programs (2). Despite this long and honored tradition, the practical clinical impact of correlating radiology and pathology is limited.
Calls for deep integrated workflows between radiology, pathology and genetics have recently increased primarily due to computational sciences technologic advances allowing a meaningful integration of these diagnostic specialties (3).

\section{NSTITUTES AND THEIR STRUCTURE}

The structure to accomplish the desired integration of clinical disciplines in many United States AMCs is that of an Institute. Institutes are patient rather than physician centered and are typically based in a disease, such as cancer, or an organ, such as heart or brain. Some salient examples of Institutes with considerable prestige and years of tradition are the Neurological Institute of New York, affiliated with Columbia University and founded in 1909 (4), and the Dana Farber Cancer Institute, affiliated with Harvard Medical School and founded in 1947.

By definition, Institutes are constituted by more that one Department integrating all the Departments dealing with its particular theme. For instance, a Neurological Institute will include in its core the traditional Departments of Neurology, Neurosurgery and Psychiatry, plus subspecialists from other Departments such as neuroradiologists, neuropathologists, neuroanesthesiologists, neurogeneticists, etc. Therefore, Institutes are centered on the patient, and thus care is enhanced in all aspects of the organ or disease of interest for regardless of the specialty of the different providers and resulting in quality improvements (5).

Institutes are organized by Centers of Excellence (COEs) dealing with relevant aspects involving the organ or disease to which the Institute is dedicated (6). For instance, typical COEs of a Neurological Institute include: 1) Brain Neoplasms, 2) Movement Disorders, 3) Stroke and Aneurysms, 4) Pituitary Disorders, 5) Eye, Ear and Skull Base, 6) Hydrocephalus, 7) Brain Health, etc. (4). 
Further, Institutes are characterized by having a single clinical and administrative leadership and therefore a single strategy, marketing plan and budget. Its single focus makes Institutes efficient and valuable, making its proliferation commonplace as stand-alone or within AMCs or other large medical centers. In either case, traditional physician-centered Departments continue to operate to ensure specialty-based training and research programs (5).

\section{DIAGNOSTIC INSTITUTE}

Considering the increasing success of disease and organ based Institutes, the integration of the core diagnostic disciplines has started to draw interest by health care systems' administrators and practicing diagnosticians alike. Establishing a Diagnostic Institute makes sense since it has the potential on one hand to increase patient satisfaction and quality of service delivered and on the other, to be innovative and bring market differentiation. In short, a Diagnostic Institute brings value, which is the Holy Grail in health care nowadays, since its establishment may increase quality while decreases cost.

A Diagnostic Institute rides not only the new wave of integration in healthcare but also takes advantage of today's other hot topic, the application of Artificial Intelligence (AI) in clinical care. Diagnostic disciplines with their immense digital data banks consisting of medical images, anatomic and clinical pathology (laboratory) information and genomics constitute the ideal field to apply AI, machine learning and big data analytics (1).

With the advent of digital pathology, the playing field for cross-disciplinary information technology (IT) tools greatly expands. Moreover, there is a strong trend for quantification of image contents to enable large-scale computational analysis. This is equally applicable for pathology and radiology, and in the latter case, it is known as radiomics (3). The disciplinary border actually becomes blurred and irrelevant when computational approaches, such as deep learning, are applied to quantify imaging features - the computational methods are the same regardless of the data source. In addition, the possibility of combining radiologic and pathologic imaging in machine learning approaches is a particularly promising aspect of ID (1)

Therefore, advances in IT provide the technological background for meaningful integration of diagnostic data and allow the take off of clinically feasible ID.

\section{DEPARTMENTS OF RADIOLOGY, PATHOLOGY AND GENETICS IN THE UNITED STATES}

To understand the many facets of Diagnostic Institutes it is essential to become familiar with its organization overview based on the typical structure of $\mathrm{Ra}$ diology, Pathology and Genetics Departments in US AMCs (Figure 1).

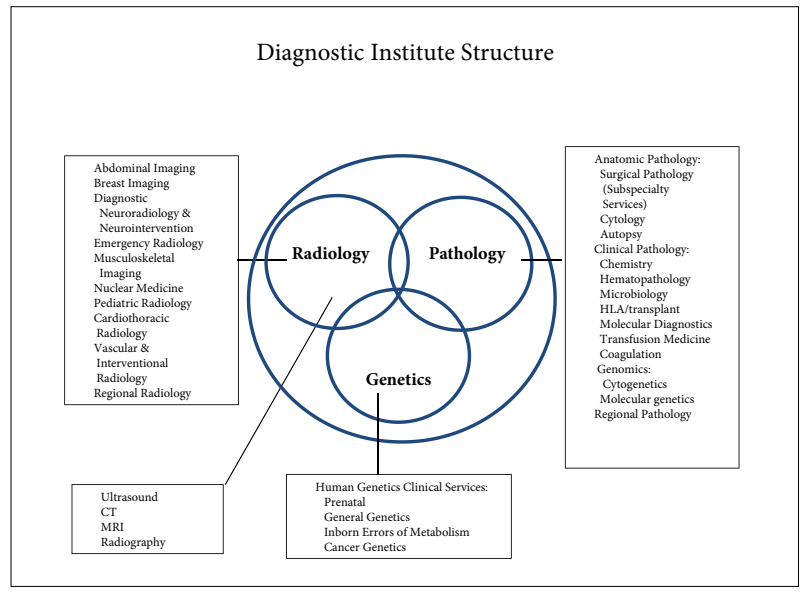

Figure 1. Example of the organizational structure of a Diagnosis Institute in an Academic Medical Center in the US. Note the Departments of Radiology, Pathology and Genetics are divided in organ, technology or disease based Divisions, Sections and in some situations Centers.

Departments of Radiology are typically organized in Divisions based on organ systems, such as: Abdominal Imaging, Neuroradiology, Breast Imaging, Vascular, Musculoskeletal and Cardio-Thoracic Radiology. Divisions can be also based on patient's age, such as Pediatric Radiology; type of service, such as Emergency Radiology, Oncoradiology and Community or Regional Radiology; and intrinsic nature (Interventional and Diagnostic Radiology). In addition, Radiology is also divided in technology-based modalities, such as Radiography or plain films, Ultrasonography, Computed Tomography, Nuclear Medicine, Magnetic Resonance Imaging and Angiography (7).

Departments of Pathology in the United States and Canada are very complex since include, along with Anatomic Pathology, the laboratory services of the medical center (8). The traditional divisions are: 1 ) Anatomic Pathology, 2) Clinical Pathology (Laboratory Medicine) and more recently, 3) Genomics and 4) Community or Regional Pathology. Anatomic Pathology is subdivided in: 1) Surgical Pathology Subspecialty Services (i.e. Neuro, GI, Breast, Cardiac, Bone, Uropathology, etc.), 2) Cytology and 3) Autopsy. Clinical Pathology or Laboratory is subdivided in: 1) Chemistry, 2) Hematopathology, 3) Microbiology, 4) Human Leukocyte Antigen (HLA) / Transplant, 5) Molecular Pathology, 6) Transfusion Medicine, 7) Coagulation. Genomics is subdivided in: 1) Cytogenetics and 2) Molecular Genetics

Departments of Genetics have a Clinical Division, frequently subdivided into: 1) Prenatal, 2) General Genetics, 3) Inborn Errors of Metabolism and 4) Cancer Genetics.

\section{CENTERS OF EXCELLENCE IN A DIAGNOSTIC INSTITUTE}

As above mentioned, Institutes are structured based on COEs. This concept is easy to understand in disea- 
se or organ oriented institutes since the COEs coordinate the efforts of specialists of different departments dealing with specific portions (spine, brain, sellar region, etc.) or diseases (oncology, degenerative, seizures, etc.) of an organ system (6)

In a Diagnostic Institute, the first COE typically established is that of Cancer Diagnosis, since in many Departments of Radiology, Pathology and Genetics there already are Divisions or Sections focused in this key disease. There may be other disease based COEs in Diagnostic Institutes devoted to diseases where diagnosis by imaging, pathology and genetics benefits by a coordinated approach by specialists. COEs for Neuro Degenerative, Cardiometabolic, Immunologic and Pediatric Diseases are after the Oncologic Diagnosis COE, frequently founded in Diagnostic Institutes. In general, once the concept of ID is embraced by a healthcare organization it is natural to develop COEs paralleling existing Institutes. For instance, if an integrated healthcare organization has Neurological, Cancer, and Gastrointestinal Health Institutes, the Diagnostic Institute will develop COEs dedicated to mirror the existing Institutes in support of the healthcare system major areas of interest (3).

Besides disease based COEs as the ones mentioned, there are others in Diagnostic Institutes. It makes sense to establish COEs centered in Population Health, Innovation, Diagnostic Optimization and Computational Sciences.

\section{CANCER DIAGNOSIS COE AND INTEGRATED TUMOR BOARDS}

Integration of radiological and pathological images, and thus achieving and integrated rad-path or diagnostic report as is done in Tumor Boards, is the most common manifestation of ID. Establishing a Diagnostic Institute provides the opportunity to expedite the electronic integration of radiological and anatomic pathology specimen images. Through single, already commercially available PACS workstations, images can be displayed side-by-side and electronically linked, resulting in the so-called Integrated Tumor Board workstation (Figure 2). This is commonly one of the earliest and most successful initiatives of a Diagnostic Institute. The Integrated Tumor Board allows identifying and reconciling discordances between radiologic and pathologic findings in every case where radiological and surgical pathology images are performed, and not only in the handful of cases presented in the Tumor Boards. Also, a reconciled, integrated report is issued and stored in the patient's electronic medical record in all concordant cases.

The final disposition of the case is linked to artificial intelligence (AI) engines for best management, potential steerage of patients to clinical trials and automatic monitoring of the follow up images for response. Clearly, an Integrated Tumor Board improves quality and reduces risks. Of the different Tumor Boards the most advanced in the adoption of an ID approach is that of Breast Cancer.

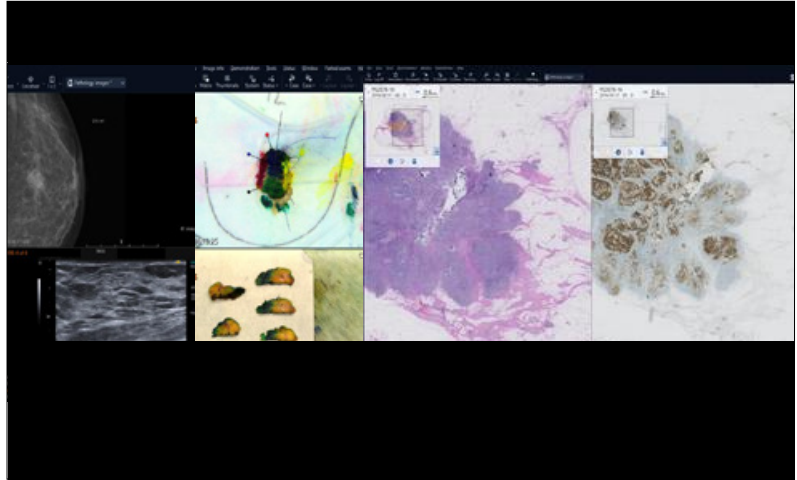

Figure 2. Integrated Diagnosis Workstation: In the same PACS workstation, mammography and breast ultrasound images are displayed integrated with surgical drawings, macroscopic specimen section and histology whole-slides in H\&E and RE. Image courtesy of SECTRA

\section{POPULATION HEALTH COE}

Radiology, Pathology and Genetics participate in screening programs of healthy population. The opportunity provided by a Population Health COE within a Diagnostic Institute is to offer Integrated Health Screening combining imaging, laboratory and genetic testing. This testing is age and gender tailored and includes screening for the top 25 genetic defects, laboratory key screening indicators, such as tumor markers and lipid panel, and imaging. Imaging screening of populations has blossomed and currently the following tests are insurance and US government approved: mammography, calcium scoring, CT colonography and lung cancer screening. Others imaging screening tests are considered outof-pocket expenses, such as breast and prostate cancer screening fast MRI scans and ultrasound scans for aortic aneurysm and liver steatosis. Both ends of the health care financial spectrum such as, executive physical exams programs and Accountable Care Organizations (ACOs) are interested in exploring integrated health screening programs. The reasons are opposed since in the first case is market differentiation, and in the latter, cost savings.

\section{DIAGNOSTIC TEST OPTIMIZATION COE}

Appropriate test utilization is of paramount importance for radiologists, pathologists and geneticists, since there is a clear tendency to conserve resources and decrease the cost of diagnosis. In Radiology there is already a long tradition of using Decision Support Systems (DSS) based upon agreed appropriateness criteria put forward by organizations such as the American College of Radiology (ACR). There is no such tradition for pathology (laboratory) and genetics, but there is interest in forming Diagnostic Testing teams composed by expert clinical pathologists and clinicians, to explore AI applications for laboratory test optimization rather than DSS. 


\section{INNOVATION COE}

Integrated Diagnosis affords a myriad of innovative ideas generated on a daily basis. A natural one is the establishment of a Molecular Diagnosis Program integrating the existing and already individually successful programs of molecular genetics, molecular pathology and molecular imaging. Another innovative idea is to integrate the image quantification efforts occurring in the three disciplines. The creation of hybrid disciplines such as Radiomics and Radiogenomics combine in a systematic fashion the core diagnostic specialties.

\section{COMPUTATIONAL SCIENCES COE}

Since the basis of ID and thus of a Diagnostic Institute is the current computational revolution it is natural to focus on a COE dedicated to Computational Sciences programs: 1) applications of AI to Diagnostics, 2) integration of diagnostic analysis of Radiology and Pathology images and reporting systems, and 3) development of analysis, query and autonomous learning systems to enable crossdisciplinary research and data pattern recognition (1).

\section{BARRIERS FOR INTEGRATED DIAGNOSIS}

The reluctance to embrace a Diagnostic Institute as a vehicle for an ID rests primarily in the resistance to change by many physicians from radiologists, pathologists and referring physicians and sometimes hospital administrators. Fear to workload increases, commoditization of radiology and pathology reads and a thread to lose management prerogatives by some referring physicians are frequently mentioned.

\section{CONCLUSION}

We believe the integration of the core diagnostic specialties of Radiology. Pathology and Genetics in the form of a Diagnostic Institute offers a great opportunity, recently made possible due to technology advances, mainly in computational sciences. The integration of diagnostic information results primarily on better patient care and brings value lowering costs. In addition, affords unique collaborations in novel research and educational programs.

\section{BIBLIOGRAFÍA}

1. Lundstrom CF, Gilmore HL, Ros PR: Integrated Diagnosis: The Computational Revolution Catalyzing Cross-Disciplinary Practices in Radiology, Pathology and Genomics. Radiology 2017; 285:12-15

2. Murphey MD, Madewell JE, Olmsted WW, Ros PR, Neiman HL\{ A History of Radiologic Pathology Co- rrelation at the Armed Forces Institute of Pathology and Its Evolution into the American Institute for Radiologic Pathology. Radiology, 2012; 262: 623-634

3. Sorace J, Aberle DR, Elimam D, Lawvere S, Tawfik $\mathrm{O}$, Wallace WD. Integrating pathology and radiology disciplines: an emerging opportunity? BMC Med 2012; 10: 100

4. Stump, E: Columbia's Neurological Institute Celebrates its Centennial Neurology Today 2009; 9, 23: 1-4

5. Braunwald, E: Departments, Divisions and Centers in the Evolution of Medical Schools. AM J Med 2006; 119, 6: 457-462

6. Elrod JK, Fortenberry JL. Centers of excellence in healthcare institutions: what they are and how to assemble them. BMC Health Serv Res 2017; 17(Suppl 1): 425 .

7. Muroff LR: Implementing an effective organization and governance structure for a radiology practice. JACR 2004; 1,1: 26-32

8. Sanfilippo F, Burns KH, Borowitz MJ, Jackson JB, Hruban RH. The Johns Hopkins Department of Pathology Novel Organizational Model: a 25-year-old ongoing experiment. Acad Pathol 2018; 5.

\section{DECLARACIÓN DE TRANSPARENCIA}

El autor/a de este artículo declara no tener ningún tipo de conflicto de intereses respecto a lo expuesto en la presente revisión.
Si desea citar nuestro artículo:

Ros-R. P.

Integrated Diagnosis: Early Experience

ANALES RANM [Internet]. Real Academia Nacional de Medicina de España; An RANM - Año 2019 • número 136 (02) · páginas 99-102 DOI: 10.32440/ar.2019.136.02.rev01 